\title{
Delay minimization and Vehicle velocity based Traffic Control Scheme in VANET
}

\author{
Namrata Mishra \\ M.tech Student \\ OIST Bhopal (M.P)
}

\author{
Sandeep Pratap \\ Dept. of computer science \\ OIST Bhopal (M.P)
}

\begin{abstract}
In Vehicular Ad Hoc Networks (VANET) networks, vehicles are equipped with communication equipment that allows them to exchange messages with each other in Vehicle-to-Vehicle communication ( $\mathrm{V}$ to $\mathrm{V}$ ) and with a roadside network infrastructure i.e. Vehicle-to-Roadside Unit Communication (V to RSU). One of the critical issues is consists of the design of delay minimization algorithms that are robust to frequent path distractions caused by vehicles mobility. The scheme presented in this paper is completely based on for reduces the delay on network for vehicles that has high velocity and arrival time in network to reduces the possibility of collision and also uses the low velocity vehicles to deliver the road status and velocity information of high velocity vehicle deliver in network for remaining vehicles and RSU and also reduces the overall traffic in highly mobile VANET networks.. The basic concept behind the proposed scheme is to broadcast only specific and well-defined packets, referred to as delay aware information to all vehicles in this paper. The performance of the scheme is analyses through computer simulations through ns-2 simulator. Simulation results indicate the benefits of the proposed routing strategy in terms of increasing delivery information, reducing the number of packets overhead and increasing the throughput.
\end{abstract}

\section{General Terms}

TCP, UDP, Synchronization Algorithm, Delay.

\section{Keywords}

VANET, RSU, AODV, Velocity.

\section{INTRODUCTION}

Vehicular ad hoc network (VANET) is a vehicle to vehicle (Inter-vehicle communication-IVC) and roadside to vehicle (RVC) communication system. The technology in VANET combines WLAN/cellular and Ad Hoc networks to achieve the continuous connectivity. The ad hoc network is put forth with the novel objectives of providing safety and comfort related services to vehicle users [1]. Some major safety related services addressed by VANET are Collision warning, traffic congestion alarm, lane-change warning, road blockade alarm (due to construction works etc.). In the other category of comfort related services, vehicle users are provide with Internet and Multimedia connectivity. We aim here to study the efficacy of Communication network in VANET on the basis of a predictable mobility model.

Beside with the recent developments in the VANET field [2], a number of striking applications, which are exclusive for the vehicular setting, has emerged. VANET applications include onboard active safety systems that are used to assist drivers in avoiding collisions and to coordinate among them at critical points such as intersections and highway entries. Safety systems may intelligently broadcast road information, for example incidents, real-time traffic congestion, high-velocity tolling, or surface condition to vehicles in the vicinity of the subjected sites. This helps to evade platoon vehicles and to accordingly improve road capacity. With the help of such active safety systems, we can expect to be largely reduced in the number of car accidents and associated damage. In addition to the aforementioned safety applications, IVC communications also used to provide comfort applications. After some time they may include information about weather, gas station or location of restaurant, mobile e-commerce, infotainment applications, and interactive communications like Internet access, music downloads, and content delivery. In this paper, we focus more on the provision of such entertaining applications.

\section{VANET ARCHITECTURE}

To provide the facility of communication between the vehicles and vehicle to RSU the wireless access medium (wave) is used. With the help of this type of communication method derivers and travelers can achieve various type of information. Communication method also provides some safety related application in order to provide the enhancement to road safety and also ensure to give the comfort driving. Onboard units (OBU), Road side unit (RSU), application unit (AU) are the components of VANET architecture are.

The RSU provide services by hosting an application and OBU is a mounted device which use the services provided by the RSU.the device which provide services is termed as provider and the device which use the application is called user the OBU is associated with each vehicle. It contain no of sensors to collect the information and to process them. With the help of wireless medium the information can be broadcast to other vehicles and to RSU. There may be single or multiple application units which use the services provide bye the provider with the help of OBU connection characteristics. If the RSU is connect to the internet it can also allow to AU from multiple vehicles to connect to internet. [6]

\section{WA VE/802.11P PROTOCOL}

To provide the communication between vehicles the IEEE standardization provides a communication environment is called WAVE. The technology for this standardization is dedicated short range communication. Which is present in IEEE 1609 standard adjusted for lower head operation.DSRC is used to improve the public safety application and to improve the traffic flow in $\mathrm{v} 2 \mathrm{v}$ in $\mathrm{v} 2 \mathrm{I}$ communication. The VANET protocol stack is approved by U.S. federal communication commission (FCC) under the dynamic short range communication (DSCR) concept.

The communication between the vehicle to infrastructure can be possible with the help of WAVE (Wireless Access in 
Vehicular Environment)[3]. It is set of standard which enable the vehicle for such type of communicationThe architecture of WAVE is composed by stacks, with common layers, as shown in Fig. 1.4.4.

\begin{tabular}{|c|}
\hline Resource Manager (IEEE 1609.1) \\
\hline \hline Security Service (IEEE 1609.2) \\
\hline \hline UDP/TCP \\
\hline IPv6 \\
(IEEE 1609.3) \\
\hline LLC (IEEE 802.2) \\
\hline Multi-Channel Operation (IEEE 1609.4) \\
\hline \hline IEEE 802.11 p \\
\hline
\end{tabular}

Figure 1 WAVE Protocol architecture

Vehicular based communication $802.11 \mathrm{p}$ was considered for dedicated short range communication (DSRC) for the international organization for standardization a U.S. Department of Transportation project. It is used particularly for some applications such as safety related services and the toll collection.

\section{PROBLEM DEFINATION \& RELATED WORK}

In the previous work, the data delivery can be achieve by only high speed vehicles with minimized delay .No further data delivery is made by slow speed vehicles. Whereas an efficient data delivery is also an important factor with minimized delay. If the slow speed vehicles carry some data but if the packet has been discarded, and that vehicle resides on road can create the collision condition in vehicular environment.

We proposed a scheme that reduces the delay possibility in heavy vehicular environment. This will identify the behavior of vehicles on the bases of their mobility in network. The slower velocity vehicle participate in the data forwarding of fast driver to provide free roads for it and RSU maintains the arrival time of each vehicle. We also try to Analyze the delay at each stationary (RSU units) and summaries overall delay.

This section has presents the previous work so far has been done in VANET velocity based delay control mechanism. This work was providing the idea about innovative work proposed in this field.

[1] In this paper they propose a scheme for Vehicular Intermittently Connected Networks (VICNs) .proposed scheme is also called Delay-Aware Data Delivery (DADD) scheme. In this scheme the stationary road side unites allow the transmission of data packets including where retransmission is required this is possible by the newly entered vehicle in its communication range. This vehicle will give the guarantee about to minimizes the delay and the retransmission of bundles and give the assurance to deliver the data to the destination SRU.the performance cane be evaluate through the mathematical model which can give the resulting bundle delay. To evaluate the performance of the proposed scheme the simulations are performed in ns also the results are compared with the two existing schemes.

[9] In this paper they discus, a scenario named Vehicular Intermittently Connected Network (VICN) is considered where point to point link is used to connect a metropolitan area network and a cell site. Because of the increment of mobile computing devices which are connected to the internet, are consume the data heavily per devices. Such as microwave links are used to transmit the large amount of data. Due to this the network faces the problem of the bandwidth insufficient of frequency.

[10] In this paper they on communication protocol which can define how the vehicle acess the wired backbone network to use of multi hop data delivery services. To address the mobility issues the cross layer protocol is designed. The cross layer design gives the permission for exchanging the information with the help of sharing of cross layer borders. Which enable the well organized and robust Protocol. There is a PROMPT named cross layer designed delay aware protocol. It has source routing mechanism which is independent from the vehicles movement besides specific value address. The PROMPT communication protocol helps to reduce the negative effect of high mobility nodes

[11]In this paper they discuss, radio overlapping a novel density model .The signalized junction points and the road segments which are linked to the junctions are the two main factors the urban traffic environment. A fundamental mechanism is proposed for the perspective of message communication a derived radio overlapping model is applied. It provides the real framework to evaluate the performance and to design the analytical framework to identify the other aspects of the data. An experimental result shows the transmission power and with the highly traffic network which reduces the channel load according to the data rate.

[12] In this paper they discus about how the information is collect of traffic by roadside unit and how to broadcast them to achieve safe communication in vanet. RSU plays an important role in such type of communication. Where the information is collect by all the vehicles. Then it has to broadcast to all those vehicles which are in range with the help of mutual cooperation of vehicles. It gives the services to drivers and helps the drivers to choose appropriate route so that he can be safe from the traffic jam condition. In this paper they also give the comparison of many existing schemes. At the end the goal is to achieve the improvement in road safety and reduce the traffic congestion problem.

[13In this papers they about some application of VANET. The vehicular adhoc network provide us the wide variety of inter vehicle communication and vehicle roadside communication from the future perspectives. With the help of various communication channels which are based on IEEE $802.11 \mathrm{p}$ to give support the wide range communication channel to acess the safety applications. Six communication channels are used for other applications. With the help of analytical model the performance can be evaluate for the safety message delivery of vehicles.

[14] In this paper they propose a system about abiding geocast by which a warning message can be broadcast in VANET. There are some strategies which can give the improvement in efficiency of broadcasting. Where the vehicles are not in same direction then the relays which has preference can overcome the fragmentation and can reduce the broadcasting. The abiding geocast if there is a problem area for vehicles it can suggest the vehicle they should left the lane as soon as before they cross the safety line. If there is a possibility vehicles can also broadcast some messages. 
[15] In this paper they discuss, suppose some people want to find the content of their interest on the road it can be possible through the vehicle to vehicle communication. But there is some limitations about contact duration time and some wireless connections which are unreliable. If the vehicle has matching data then only it can acess the useful data. a novel point to point sharing scheme is proposed for give the improvement in the performance of content sharing scheme. Which focus on the user query requirement so that it has so many chances to request about content data. There are two major components in the road cast.

\section{PROPOSED WORK}

In the proposed delay aware scheme based on V to RSU. The data is transferred to Vehicles for aware about the traffic information is included in the control messages. The delay minimizing mechanism is proposed in this paper, the RSU has to know about the vehicles parameter in order they are arriving to process the data and to release the data properly. So that the first connection establishment is required between the RSU and newly arrived vehicle. To establish the connection vehicle send the request when it sense the presence of RSU. And also give the information about itself like its speed and its arrival time. This connection establishment process is very fast and instantaneously performed. The RSU is the main server which controls the all operations for communication. The algorithm for proposed mechanism contains some steps which are as follows:

1) Set the RSU unit at junction points.

2) There are n no. of vehicles, RSU send connection setup request to neighbour vehicles. Vn vehicles send their info such as Velocity, location and start time.

3) RSU receives their info and store in database for synchronisation, also monitor all the vehicles.

4) When $\mathrm{Vi}$ to $\mathrm{Vi}+\mathrm{j}$ vehicles reach at RSU and the no. of vehicles are more than two , than RSU capture the information and hold the vehicle for some time to perform the synchronisation with in a interval .

5) While vehicle has high speed, synchronisation the vehicles, no. of synchronised vehicles should be more than 3.

6) Send the higher priority data with high speed vehicle and departure the vehicle .decrease the queue value by 1 .

7) Eliminate vehicles from $V_{I}$ to $V_{j}$ and set $V_{i+j}$ as low velocity. Send the lower priority data with low speed vehicles.

8) If there is no congestion problem, we can easily give stop time to all vehicles and send the data.

9) RSU can also communicate with each other if they have a mutual communication range

10) Analyse the each RSU and delay aware info of each vehicle.

\subsection{Proposed working Algorithm for \\ VANET}

Set RSU's: Road Side Unit

$$
\text { Set } \mathrm{V}=\left\{V_{i}, \mathrm{~V}_{\mathrm{i}+1}, \cdots-V_{i+j}, \cdots V_{n}\right\} \quad / / V_{n} \text { Vehicle's }
$$

RSU's Send Hello Message to all neighbour station. $v_{n}$ Vehicles' sends info (velocity, location, start time)

\section{RSU receives info (vehicle)}

Store info in data base for synchronization and watching the $V_{n}$ Watch all $\mathrm{V}_{\mathrm{i} \text {------- }} V_{n}$ vehicles in particular route.

If $\left(V_{i} \cdots V_{i+j}\right.$ arrive at $\mathrm{T}$ station $\& \& V_{i}>2$ in $\left.\mathrm{T}\right)$

\{

Capture all $V_{i} \cdots V_{j}$ information at delay $\partial \mathrm{t}=5$ as a stop every $30^{\text {th }} \min$

Synchronize $V_{i} \cdots V_{i+j}$ on the bases of velocity of vehicle

While $\left(V_{i+j}\right.$ velocity $==$ high $\& \&$ resynchronized $\left(V_{i}\right.$ to $V_{j}>=$ 3))

\{

Send higher priority data;

Departure the $V_{i+j}$;

Decrease queue by 1 ;

)

Set $V_{i \text { to }} V_{j}$ eliminate $V_{i+j}$ as low velocity

Send lower priority data;

Departure the vehicle;

\}

// No Congestion Case

Else

\{

Stop time is 1;

Send data'

\}

If (RSUs In range)

\{

Communicate $\mathrm{RSU}_{\mathrm{K}}$ to $\mathrm{RSU}_{\mathrm{L}}$

\}

Analyze each T stations and delay aware of each vehicle

Analyze data at end station

Stop;

\section{SIMULATION ENVIRONMENT}

There is a technique to solve the problems by the observation of the performance, with the time, of a dynamic model of the system is called simulations." [52]. Simulation generally illustrates the relationship between the systems and models. A system is the collection of components that are interrelated and interacted in such a way that it distinguishes the system from its environment. A model is a "simplified illustration of the system intended to predict what happens if certain actions are taken" [52]. Simulation development is iterative process in which the construction, execution and analysis of a model repeatedly perform in order to achieve desired level of understandings [53]. A simulation process is shown in figure . 


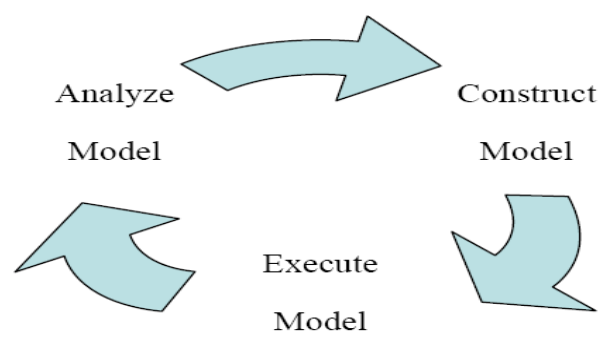

Figure 2: Modeling process [33]

\subsection{Simulation Tool/ Simulator}

For the research work in communication network the simulator is designed which is an open source event driven. This resembles with network simulator which also called ns2.the evaluatation of the performance of routing protocol is the main working of simulator from Berkeley. The mobility extensions are used to $\mathrm{ns}$ for simulation in the radio environment of mobile wireless.

There are some scripting languages which are used to evaluate the performance of network and also to configure the network. And results are evaluated by NS2. NS2 is the most used open source network simulator.

Table 1 Simulation Parameters

\begin{tabular}{|l|l|}
\hline Number of nodes & 100 \\
\hline Dimension of simulated area & $800 \times 800$ \\
\hline Routing Protocol & AODV \\
\hline RSU unit & 4 \\
\hline Simulation time (seconds) & 100 \\
\hline Transmission Range size & $250 \mathrm{~m}$ \\
\hline $\begin{array}{l}\text { Information Packet } \\
\text { (bytes) }\end{array}$ & 512 \\
\hline Maximum Speed (m/s) & 30 \\
\hline Nodes Mobility & Random way point \\
\hline
\end{tabular}

\section{SIMULATION RELULTS}

The simulation results on the basis of proposed scheme are mentioned in this section. The results are clearly shows that the better performance in proposed delay based.

\subsection{VANET Traffic Scenario}

This network animator (nam) scenario represents the VANET scenario of vehicles. In this scenario the four RSU units are considered and all the vehicles are monitored by these RSU units and every RSU unit contain the information of every vehicle i.e. arrival rate, velocity of vehicle.

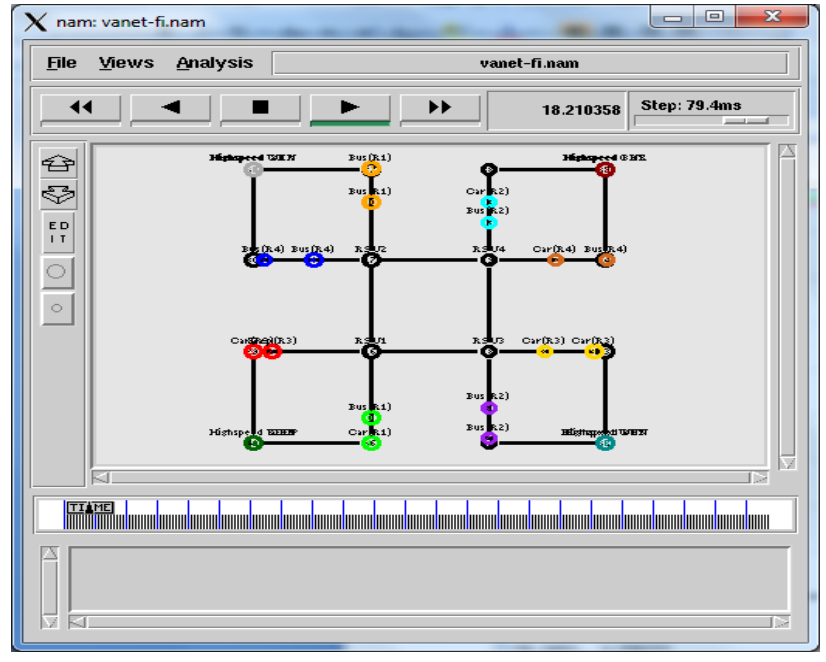

Fig 2: VANET NAM Scenario

\subsection{TCP data traffic at destination in Previous VANET Scenario}

This graph represents the performance of TCP (Transmission Control protocol) data packets traffic in case of previous AODV routing.tcp is connection oriented protocol that's why it is also called reliable protocol. It means this protocol is time consuming because of sender first establish connection with receiver then start data delivery and if receives acknowledgement from receiver then again same procedure is call again. This graph show the TCP congestion window of five connections and the size of congestion window are normal and provide better data delivery in network. The main drawback of this normal packet delivery size of congestion window is large only at start of simulation about time 70 to 100 minutes, by that the possibility of data packet dropping and acknowledgement dropping are more and the delay and collision is more.

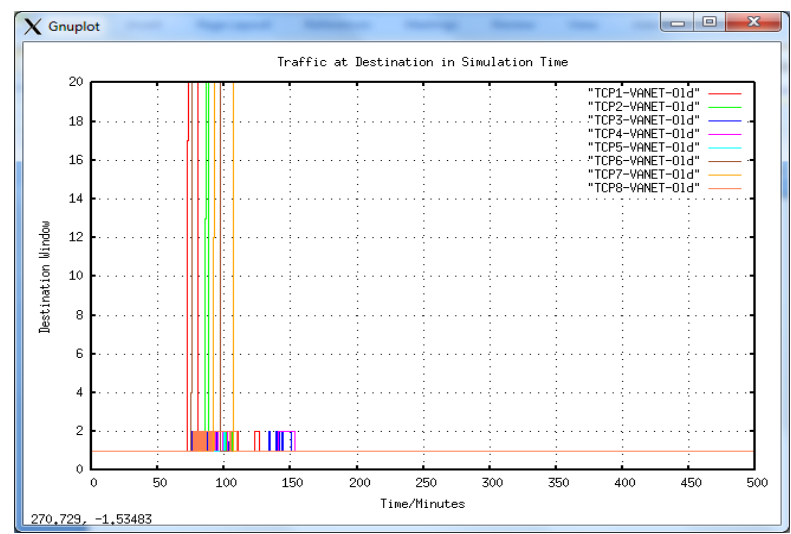

Fig 3: Previous TCP Traffic Analysis

\subsection{Proposed TCP data traffic at destination in Normal VANET}

This graph also represents the TCP packet performance in case of Updated AODV routing. Here notice the packet transmission of connection TCP1. The TCP congestion window size is maximum at time in between 50 to 150 minutes. But if notice same connection in normal then after first transmission negligible packets are deliver in network. The all TCP connections has very large congestion window in 
normal case but here the size of congestion window is normal by that the packet drooping are minimize and the packet receiving possibility increases. The performance of rest of connection as also improves by applying proposed delay scheme to maintain the organization in between slow and fast vehicle.

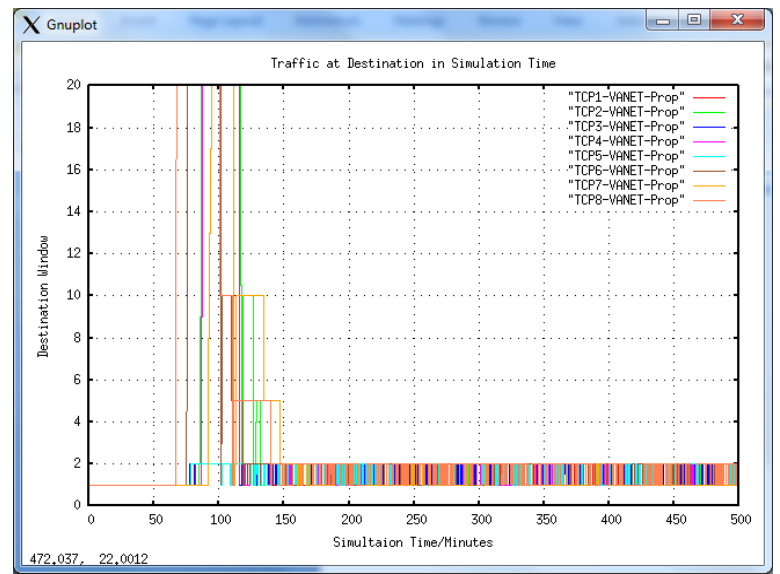

Fig 4: Proposed TCP Traffic Analysis

\subsection{Slow Traffic Transmission}

UDP (User Datagram Protocol) are providing the data delivery continuously without accepting any acknowledgment from receiver by that this protocol is not reliable for communication. But if the network performance are better means not heavy traffic, free from attacker and handle load efficiently then the performance of UDP protocol are also better. In this graph we measure the UDP packet performance in case of Normal AODV routing with slow traffic transmission. Here we clearly notice that the performance of UDP packet transmission, receiving and loss. The loss rate with respect to time is very low and the data deliver is at time about 200 minutes are equal to transmission but after that the data is lost because of take part of more vehicle in network. Now the performance are shows that the delay is minimizes and reduces collision also.

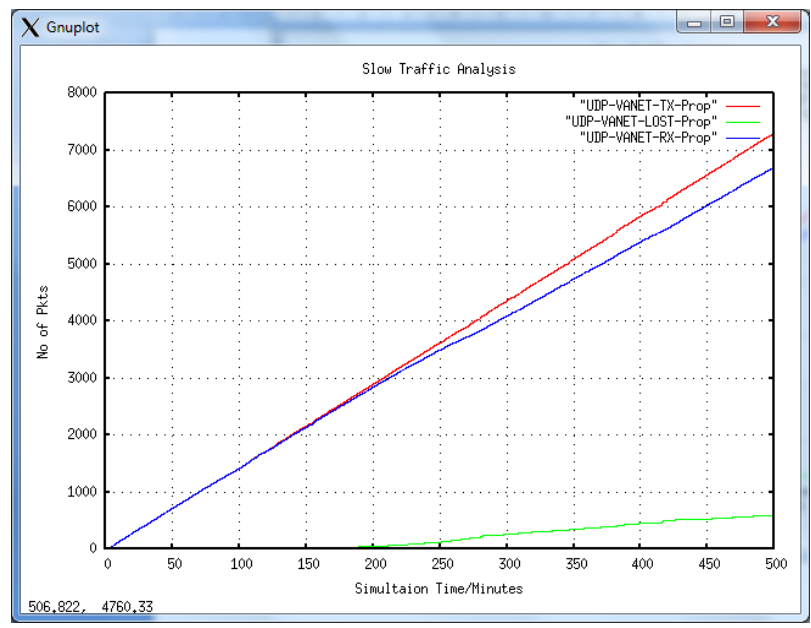

Fig 5: UDP Proposed Traffic Analysis

\subsection{Control Message}

Control packets are required in network to maintained connection in between sender and receiver for reliable traffic information. The control packets are first discover the route on the basis of routing procedure of routing protocol like AODV and if the receiver are send back to so sender connection confirmation packets then the data delivery is started in between sender and receiver. The traffic information is delivering after establishment of connection. This graph has show the control packets analysis in case of normal VANET with AODV and proposed VANET with AODV routing protocol. The difference in performance of number of control packets in both the cases is about 2500 control packet and also the data receiving in network in case of proposed scheme is more with minimum delay and collision.

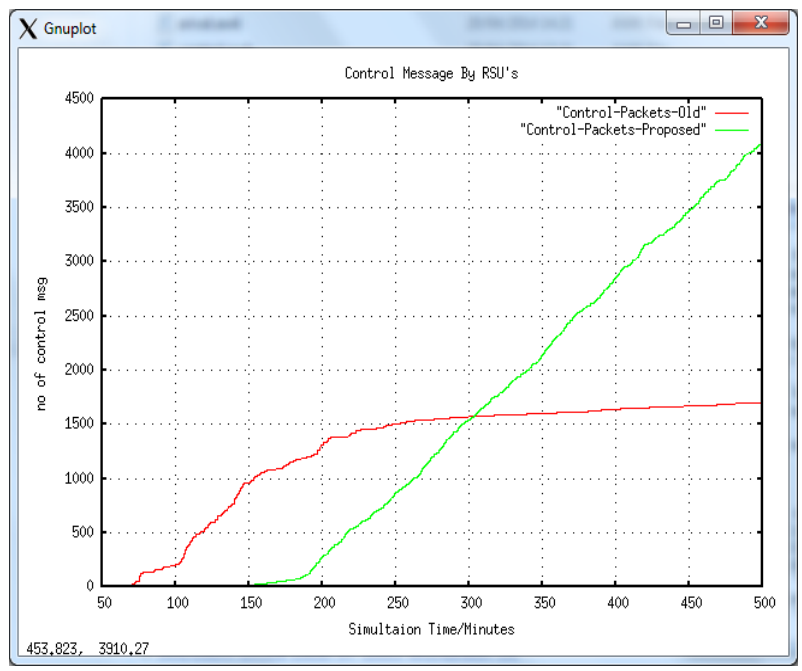

Fig 6: Control Overhead Analysis

\subsection{Successful Deliver Analysis}

The delivery percentage analysis is the ratio of successful percentage of packets that are received in network. This graph is represents the PDF analysis in case of normal VANET traffic and updated delay based velocity organization of vehicles. Here the performances of both the protocol are almost equal in time duration about 150 minutes but after that the percentage of data receiving are not counted in normal VANET with AODV routing Protocol but in proposed scheme the amount of packets in network are deliver more by that the throughput in case updated delay based VANET with AODV routing is improved and also the reliability of receiving of packets at destination are get better than normal because of minimizes the collision.

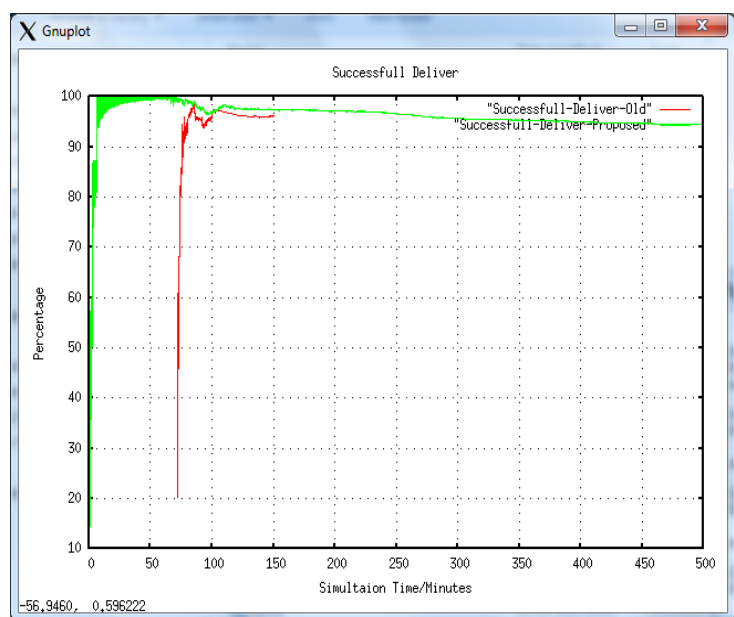




\subsubsection{Analysis of RSU1}

The RSU unit is maintaining the information of each vehicle in network. The table 3 represents the RSU 1 information in case of normal VANET scenario (OLD). In case of proposed scheme the delay of most of vehicles and collision are minimizes of node or vehicle $21,22,24$ and the vehicle is no information are not participate in routing procedure and the number of vehicle in case of proposed scheme is only 1 , but is case of normal VANET is 4. It means the utilization of about all vehicles is only in proposed scheme for traffic delay handling.

Table: 3 Analysis of RSU1

\begin{tabular}{|c|c|c|c|c|}
\hline \multicolumn{5}{|c|}{ Comparison of Arrival and Delay at RSU1 } \\
\hline Node & $\begin{array}{c}\text { Arrival } \\
\text { Old }\end{array}$ & Delay Old & $\begin{array}{c}\text { Arrival } \\
\text { Proposed }\end{array}$ & $\begin{array}{c}\text { Delay } \\
\text { Proposed }\end{array}$ \\
\hline 0 & 70 & 70 & 60.5 & 60.5 \\
\hline 2 & 85.5 & 15.5 & 80.5 & 20 \\
\hline 20 & 85.5 & 0(Collide) & 82.2 & 1.7 \\
\hline 21 & No Info & No Info & 89.3 & 7.1 \\
\hline 27 & 105.9 & 20.4 & 106.1 & 16.8 \\
\hline 3 & 102.8 & 3.1 & No Info & No Info \\
\hline 25 & 110 & 7.2 & 109.8 & 3.7 \\
\hline 26 & 114 & 4 & 112 & 2.2 \\
\hline 24 & 114 & 0(Collide) & 112.8 & 0.8 \\
\hline 22 & 143 & 29 & 142.9 & 30.1 \\
\hline 23 & 145.9 & 2.9 & 145.9 & 3 \\
\hline 10 & 232.9 & 87 & 226.4 & 12.8 \\
\hline 11 & No info & No Info & 245.6 & 19.2 \\
\hline 9 & 260.5 & 27.6 & 260.3 & 14.7 \\
\hline
\end{tabular}

\subsubsection{Analysis of RSU2}

The table 4 represents the RSU 2 information in case of normal VANET scenario (OLD). In case of proposed scheme the delay of most of the vehicles are minimizes and collision of node 11 removes i.e. exist in normal VANET scheme. Vehicle like 3,22, 14 are the vehicle having no information are not participate in routing procedure and in case of proposed scheme all the vehicles are handled traffic information. It means the utilization of about all vehicles is only in proposed scheme for traffic delay handling.

Table: 4 Analysis of RSU2

\begin{tabular}{|l|l|l|l|l|}
\hline \multicolumn{5}{|c|}{ Comparison of Arrival and Delay at RSU2 } \\
\hline Node & $\begin{array}{l}\text { Arrival } \\
\text { Old }\end{array}$ & $\begin{array}{l}\text { Delay } \\
\text { Old }\end{array}$ & $\begin{array}{l}\text { Arrival } \\
\text { Proposed }\end{array}$ & $\begin{array}{l}\text { Delay } \\
\text { Proposed }\end{array}$ \\
\hline 4 & 65 & 65 & 31.5 & 31.5 \\
\hline 24 & 92.4 & 27.4 & 92.1 & 60.6 \\
\hline 25 & 96.6 & 4.2 & 96 & 3.9 \\
\hline 21 & 106.2 & 1.8 & 106.3 & 2.1 \\
\hline 5 & 122.5 & 16.3 & 122.1 & 15.8 \\
\hline 3 & $\begin{array}{l}\text { No } \\
\text { Info }\end{array}$ & No Info & 141.7 & 7.6 \\
\hline 10 & 157 & 20 & 151.1 & 9.4 \\
\hline 11 & 157 & 0(Collide) & 152.9 & 1.8 \\
\hline 22 & $\begin{array}{l}\text { No } \\
\text { Info }\end{array}$ & No Info & 167.1 & 5.2 \\
\hline 14 & $\begin{array}{l}\text { No } \\
\text { Info }\end{array}$ & No Info & 198.5 & 31.4 \\
\hline 2 & 266 & 99 & 261.6 & 63.1 \\
\hline \multicolumn{5}{|l}{} \\
\hline
\end{tabular}

\section{CONCLUSION}

In vanet the RSU and vehicle are two major components for communication purpose. Such as the important message broadcasting about the imminent collision or about the velocity information of vehicle. The road side unit can be fixed in the area of one kilometer or less then it. So that the heavy traffic can be maintained. The road side unit can identify the velocity limit according to the internal database of traffic. Control and services packets delay are also affecting the traffic in vanet. These delays increases as well as increment in messages generation rate because of limited bandwidth and due to not pass the fast vehicles in road, this is traffic delay. In this proposed scenario the four RSU units are considered and the performance of proposed scheme is compare to normal VANET scenario. The proposed scheme is shows that collision of vehicles and the utilization of low velocity vehicles are also involve in network data forwarding and traffic arriving. The rest of the network performance in case of control message overhead, delivery ratio is improves with no collision in network.

\section{ACKNOWLEDGMENTS}

I am grateful to my Project Guide Mr. Sanjay Sharma whose guidance by we successfully completed our project. They willingly took the trouble of sparing his valuable time for our work and were always ready to help with his knowledge and valuable suggestions

\section{REFERENCES}

[1] Yue Liu, Jun Bi, Ju Yang; "Research on Vehicular Ad Hoc Networks"; Chinese Control and Decision Conference (CCDC), 2009. Page(s): 4430 - 4435.

[2] Tarik Taleb, Sakhaee, Abbas Jamalipour, , Kazuo Hashimoto, Nei Kato, And Yoshiaki Nemoto, "A Stable Routing Protocol To Support Its Services In Vanet Networks" IEEE Transactions on Vehicular Technology, Vol. 56, No. 6, pp.3337-3347 November 2007.

[3] Uzcategui, R. and G. Acosta-Marum. Wave: A tutorial. IEEE Communications Magazine. 2009, vol. 47, iss. 5, pp. 126\{133. ISSN 0163-6804. DOI: 10.1109/MCOM.2009.4939288.. 
[4] Fazio, P., F. De rango, C. Sotille and C. Calafate. A New Channel Assignment Scheme for Interference-Aware Routing in Vehicular Networks. In: 73rd Vehicular Technology Conference (VTC Spring, 2011). Yokohama: IEEE, 2011, pp. 1\{5. ISBN 978-1-424483327

[5] Fazio, P., F. De rango and C. Sotille. A new interference aware on demand routing protocol for vehicular networks. In: International Symposium on Performance Evaluation of Computer\& Telecommunication Systems (SPECTS). The Hague: IEEE, 2011, pp. 98\{103. ISBN 978-1-4577-0139-9.

[6] De rango, F., F. Gurrriero, S. Marano and E. BRUNO. A multiobjective approach for energy consumption and link stability issues in ad hoc networks. IEEE Communications Letters. 2006, vol. 10, iss. 1, pp. $28\{30$. ISSN 1089-7798. DOI: 10.1109/LCOMM.2006.1576559.

[7] De rango, F., P. Fazio and S. Marano. Utility-Based Predictive Services for Adaptive Wireless Networks With Mobile Hosts. IEEE Transactions on Vehicular Technology. 2009, vol. 58, iss. 3, pp. 1415\{1428. ISSN 0018-9545. DOI: 10.1109/TVT.2008.924989.

[8] Saif Al-Sultan n, Moath M. Al-Doori, Ali H. A Bayatti, Hussien Zedan" A comprehensive survey on vehicular Ad Hoc network" Journal of Network and Computer Applications. 2013 elsevier.jnca

[9] Mrs. A. N. Mahajan, Mrs Dimple Saproo, Mrs Rakhi Khedikar3 "analysis and comparison of vanet routing protocol using IEEE 802.11p And wave" in International Journal of Engineering Research and Applications (IJERA) ISSN: 2248-9622 www.ijera.com Vol. 3, Issue 4, Jul-Aug 2013, pp.1556-1561

[10] Maurice J. Khabbaz, Hamed M. K. Alazemi, and Chadi M. Assi, "Delay-Aware Data Delivery in
Vehicular Intermittently Connected Networks", IEEE Transactions on Communications, Vol. 61, No. 3, March 2013.

[11] Si-Ho Cha, "A Route-Aware Delay Tolerant Routing Protocol for Sparse Vehicular Ad-Hoc Networks" International Journal of Control and Automation Vol.6, No.5, pp.199-206, 2013.

[12] Ate Abdrabou, Member, IEEE and Weihua Zhuang, Fellow, IEEE "Probabilistic Delay Control and Road Side Unit Placement for Vehicular Ad Hoc Networks with Disrupted Connectivity" IEEE Journal on selected areas in communication, VOL. 29, NO. 1, January 2011

[13] Saeed Bastani, Bjorn Landfeld and Lavy Libman, "Traffic Density Model for Radio Overlapping in Urban Vehicular Ad hoc Networks", 36th Annual IEEE Conference on Local Computer Networks LCN, pp. 8592, 2011.

[14] Feng Zhang, Jianjun Hao and Shan Le "Traffic Information Aggregation and Propagation Scheme for VANET In City Environment", 2010 3rd IEEE International Conference on Broadband Network and Multimedia Technology (IC-BNMT), pp. 619 - 623 2010 .

[15] Ghada Badawy, Jelena MiSiC, Terry Todd and Dongmei Zhao, "Performance Modeling of Safety Message Delivery in Vehicular Ad Hoc Networks", 2010 IEEE 6th International Conference on Wireless and Mobile Computing. Networking and Communications, pp.188$195,2010$.

[16] Qiangyuan, Y., Geert, H., "Abiding geocast for warning message dissemination in vehicular ad hoc networks," The IEEE International Conference on Communications, Beijing, China, pp. 400-404, 2008.

[17] Web reference, http://www.isi.edu/nsnam/ns. 